\title{
A New Algorithm for Finding a Minimum Dominating Set of Graphs
}

\author{
P. Pradhan, \\ Department of Mathematics and Statistics, \\ Gurukula Kangri University, Haridwar (UK), India
}

\begin{abstract}
In the present paper the concept of relative domination power of vertices for finite undirected graphs have been introduced. An algorithm has been developed to obtain a minimum dominating set of a graph. Some results related to domination number $\gamma$ and other graph theoretic parameters for a tree also obtained.
\end{abstract}

\section{Keywords}

Dominating set, Domination number, Domination power of a vertex, Support of a graph

\section{INTRODUCTION}

All graphs considered in this paper are undirected and finite. Most of the graph theoretic notations have been borrowed from the book by Harary [1]. A graph $G=\left(V_{G}, E_{G}\right)$ consist a finite set of vertices $V_{G}$ together with an edge set $E_{G}$ such that each edge is an unordered pair of vertices. A subset $D$ of $V_{G}$ is said to be dominating set of a graph $G$ if every vertex which is not in $D$ is adjacent to at least one vertex in $D$. The domination number $\gamma$ of $G$ is the cardinality of smallest dominating set of $G$. A dominating set $D$ is said to be connected dominating set if induced subgraph $\langle D\rangle$ is connected [5]. $D$ is called independent dominating set if induced subgraph $\langle D\rangle$ is null graph. The connected domination number $\gamma_{c}$ and the independent domination number $\gamma_{i}$ of $G$ are the cardinality of smallest connected dominating set and smallest independent dominating set of $G$ respectively.

A vertex which is adjacent to pendent vertex is called support of $G$ [2]. All vertices of $G$ except pendent vertices are internal vertices. $N(v)=\left\{u \in V_{G} \mid u v \in E_{G}\right\}$ is called neighbourhood of $v$ in $G$ and the closed neighbourhood of $v$ is denoted by $\bar{N}(v)$ and is defined as $\bar{N}(v)=N(v) \cup\{v\}$. A set of vertices is said to be an independent set of vertices if no two vertices in the set are adjacent and the number of vertices in the largest independent set is called the independence number of a graph [3].

The distance between two vertices $u$ and ${ }^{v}$ of a graph $G$ is denoted by $d(u, v)$ and is defined as the length of shortest path between them.

\section{SOME DEFINITIONS}

\subsection{Definition}

A vertex $v$ in $G$ dominates another vertex $u$ in $G$ if $d(u, v) \leq 1$. Obviously $d(u, u)=0 \leq 1$, so $u$ dominates itself.

\author{
B. S. Rawat, \\ Department of Mathematics and Statistics, \\ Gurukula Kangri University, Haridwar (UK), India
}

\subsection{Definition}

A vertex $v \in V_{G}$ dominates itself and all the vertices adjacent to it, that is $v$ dominates every vertex in its closed neighborhood [4]. The number of vertices in $\mathrm{G}$ which are dominated by $v$ is known as domination power of $v$, it is denoted by $d p(v)$ so $d p(v)=|\bar{N}(v)|$.

\subsection{Definition}

The relative domination power of a vertex $v \in V_{G}$ with respect to any vertex $u \in V_{G}$ is denoted by $d p_{u}(v)$ and defined as the number of vertices which are dominated by $v$ but not by $u$. Similarly the relative domination power of a vertex $v \in V_{G}$ with respect to $u_{i}, u_{j}, \ldots, u_{k}$ in $V_{G}$ is given by $d p_{u_{i}, u_{j}, \ldots, u_{k}}(v)=$ Number of vertices dominated by $v$ but not by $u_{i}, u_{j}, \ldots, u_{k}$.

\subsection{On the Basis of Above Definition 2.3 We Have the Following Observation}

\subsubsection{Observation}

If $G$ is a tree and $d_{v_{j}}\left(v_{i}\right)=0 ; v_{j}$ is an internal vertex and adjacent to vertex $v_{i}$, then $v_{i}$ is a pendent vertex.

\subsubsection{Observation}

If for a graph $G, d p_{v_{j}}\left(v_{i}\right)=0 ; \forall v_{i}, v_{j} \in V_{G}$, then $G$ is a complete graph.

\subsubsection{Observation}

If for a graph $G, \quad v_{i} v_{j} \in E_{G} \quad$ such that $d p_{v_{j}}\left(v_{i}\right)=1 ; \forall v_{i}, v_{j} \in V_{G}$ and $\left|V_{G}\right|>3$, then $G$ is a cycle.

\subsubsection{Observation}

If for a graph $G, v_{i} v_{j} \in E_{G}$, then $d p_{v_{j}}\left(v_{i}\right) \leq \operatorname{deg}\left(v_{i}\right)-1$, $\forall v_{i}, v_{j} \in V_{G}$

\subsection{EXAMPLE}

In the following graph $d p\left(v_{1}\right)=3, d p\left(v_{2}\right)=4, d p\left(v_{3}\right)=2$, $d p\left(v_{4}\right)=2, d p\left(v_{5}\right)=4, d p_{v_{2}}\left(v_{1}\right)=1, d p_{v_{5}}\left(v_{1}\right)=1, d p_{v_{1}}\left(v_{2}\right)=2$, $d p_{v_{3}}\left(v_{2}\right)=2, \quad d p_{v_{4}}\left(v_{2}\right)=2, \quad d p_{v_{2}}\left(v_{3}\right)=0, \quad d p_{v_{1} v_{3}}\left(v_{2}\right)=1$, $d p_{v_{1} v_{3} v_{4}}\left(v_{2}\right)=0$ etc. 


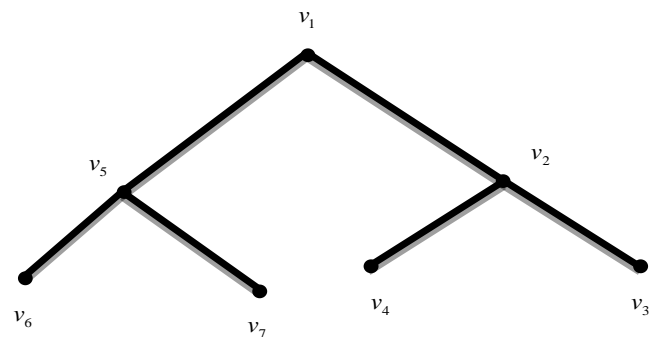

Fig 1: Tree with seven vertices

3. AN ALGORITHM FOR FINDING A MINIMUM DOMINATING SET OF A GRAPH $G$ BY USING RELATIVE DOMINATION POWER OF VERTICES Case $I$-When set of supports exist in $G$

1. Let $G=\left(V_{G}, E_{G}\right)$ be a graph of order $n$ with $t$ supports.

2. Let $S=\left\{v_{1}, v_{2}, v_{3}, \ldots, v_{t}\right\}$ be the set of supports such that $d p\left(v_{1}\right)=k_{1}, d p_{v_{1}}\left(v_{2}\right)=k_{2}, d p_{v_{1}, v_{2}}\left(v_{3}\right)=k_{3} \ldots, d p_{v_{1}, v_{2}, v_{3} \ldots v_{t-1}}\left(v_{t}\right)=k_{t}$.

3. If $k_{1}+k_{2}+k_{3}+\ldots+k_{t}=n$ and $d p_{v_{1}, v_{2}, v_{3} \ldots v_{t}}\left(v_{i}\right)=0 ; \forall v_{i} \notin S$ then $D=S$ is a minimum dominating set and domination number $\gamma=|S|$.

4. If $k_{1}+k_{2}+k_{3}+\ldots .+k_{t}<n$, then we take a set $S_{1}$ such that $S_{1}=S \cup\left\{v_{i}\right\}$; where $v_{i}$ is an internal vertex except support and having maximum relative domination power with respect to $v_{1}, v_{2}, v_{3}, \ldots v_{t}$. Let $d p_{v_{1}, v_{2}, v_{3}, \ldots v_{t}}\left(v_{i}\right)=k_{t+1}$ and $S_{1}=\left\{v_{1}, v_{2}, v_{3}, \ldots, v_{t}, v_{i}\right\}$.

Again if $k_{1}+k_{2}+k_{3}+\ldots+k_{t}+k_{t+1}=n$ and $d p_{v_{1}, v_{2}, v_{3}, \ldots v_{t}, v_{i}}$ $\left(v_{j}\right)=0 ; \forall v_{j} \notin S_{1}$. Then $D=S_{1}$ is a minimum dominating set and domination number $\gamma=\left|S_{1}\right|$.

5. If $k_{1}+k_{2}+k_{3}+\ldots+k_{t}+k_{t+1}<n$, then the process as given in step 4 is continued in similar manner until

$$
\sum_{1 \leq i \leq n} k_{i}=n
$$

\section{Case II -When set of supports does not exist in $G$}

If a graph does not have any support then in order to find a minimum dominating set for a graph; first we select a vertex $v_{i} \in V_{G}$ of maximum domination power among all the vertices of $G$.

Now if $d p\left(v_{i}\right)=n$, then the set $D=\left\{v_{i}\right\}$ is a minimum dominating set but if $d p\left(v_{i}\right)<n$, then the process as given above in step 3 and 4 is continued in similar manner until to get a dominating set.

\subsection{Example}

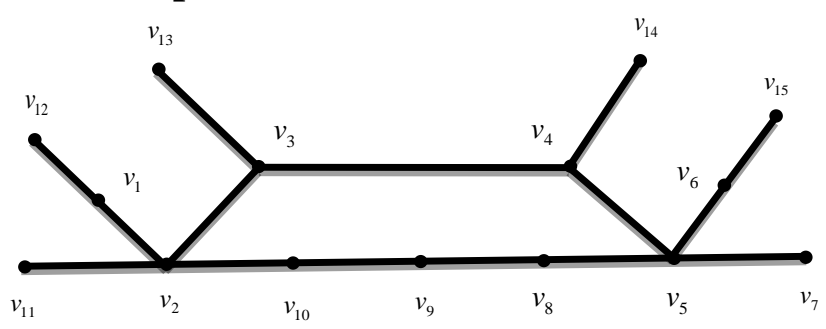

Fig 2: Graph with six supports and fifteen vertices

1. Let us consider the Fig.-2 of the graph $G=\left(V_{G}, E_{G}\right)$ of order $n=15$.

2. Let $S=\left\{v_{1}, v_{2}, v_{3}, v_{4}, v_{5}, v_{6}\right\}$ be the set containing all the supports therefore $|S|=t=6$ and $d p\left(v_{1}\right)=3, d p_{v_{1}}\left(v_{2}\right)=3, d p_{v_{1}, v_{2}}$

$\left(v_{3}\right)=2, d p_{v_{1}, v_{2}, v_{3}}\left(v_{4}\right)=2, d p_{v_{1}, v_{2}, v_{3}, v_{4}}\left(v_{5}\right)=3, d p_{v_{1}, v_{2}, v_{3}, v_{4}, v_{5}}\left(v_{6}\right)=1$ that is $k_{1}=3, k_{2}=3, k_{3}=2, k_{4}=2, k_{5}=3, k_{6}=1$.

3. $k_{1}+k_{2}+k_{3}+\ldots+k_{6}=14<n$. At this stage we have only three internal vertices except supports $v_{8}, v_{9}, v_{10}$ sucht that $d p_{v_{1}, v_{2}, v_{3}, v_{4}, v_{5}, v_{6}}\left(v_{8}\right)=1, d p_{v_{1}, v_{2}, v_{3}, v_{4}, v_{5}, v_{6}}\left(v_{9}\right)=1, d p_{v_{1}, v_{2}, v_{3}, v_{4}, v_{5}, v_{6}}\left(v_{10}\right)=1$ and we see that $v_{8}, v_{9}$ and $v_{10}$ have same relative domination power with respect to $v_{1}, v_{2}, v_{3}, v_{4}, v_{5}, v_{6}$ so we can take anyone vertex out of $v_{8}, v_{9}$ and $v_{10}$. Suppose we select $v_{9}$ then $k_{7}=1$. Hence $S_{1}=S \cup\left\{v_{9}\right\}=\left\{v_{1}, v_{2}, v_{3}, v_{4}, v_{5}, v_{6}, v_{9}\right\}$.

4. Now $k_{1}+k_{2}+k_{3}+\ldots+k_{6}+k_{7}=15=n \quad$ and $d p_{v_{1}, v_{2}, v_{3}, v_{4}, v_{5}, v_{6}, v_{9}} \quad\left(v_{i}\right)=0 ; \forall v_{i} \notin S_{1}$. Therefore $\quad D=S_{1} \quad$ is minimum dominating set and $\gamma=\left|S_{1}\right|=7$.

\subsection{Example}

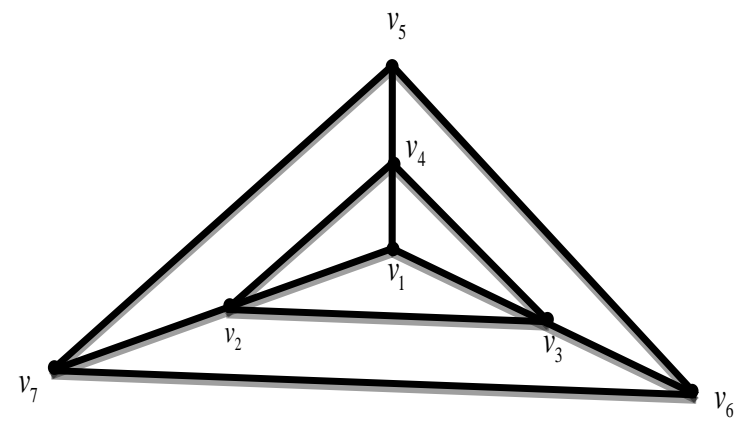

Fig 3: Graph without any support

1. Let $G=\left(V_{G}, E_{G}\right)$ be the graph as given Fig. 3 such that $n=7$ and $t=0$.

2. Since there is no support in $G$ therefore we construct the set $S$ by taking the vertex having maximum domination power.

Now $\quad d p\left(v_{1}\right)=4, \quad d p\left(v_{2}\right)=5, \quad d p\left(v_{3}\right)=5, \quad d p\left(v_{4}\right)=5$, $d p\left(v_{5}\right)=4, d p\left(v_{6}\right)=4, d p\left(v_{7}\right)=4$.

Since the vertex $v_{2}, v_{3}$ and $v_{4}$ have maximum domination power, therefore we can take any vertex out of $v_{2}, v_{3}$ and $v_{4}$ as an element of $S$.

3. Let us take $v_{2}$ in $S$ that is $S=\left\{v_{2}\right\}$ and $d p\left(v_{2}\right)=5$ that is $k_{1}=5$. 
4. Since $k_{1}=5<n$ so we construct another set $S_{1}$ by extending set $S$.

Now $d p_{v_{2}}\left(v_{1}\right)=0, d p_{v_{2}}\left(v_{3}\right)=1, d p_{v_{2}}\left(v_{4}\right)=1, d p_{v_{2}}\left(v_{5}\right)=2$, $d p_{v_{2}}\left(v_{6}\right)=2, d p_{v_{2}}\left(v_{7}\right)=2$.

Since the vertex $v_{5}, v_{6}$ and $v_{7}$ have same maximum relative domination power with respect to $v_{2}$ therefore we can take anyone out of $v_{5}, v_{6}$ and $v_{7}$ in set $S_{1}$.

5. Suppose we take $v_{5}$ in $S_{1}$ that is $S_{1}=\left\{v_{2}, v_{5}\right\}$ and $d p_{v_{2}}\left(v_{5}\right)=2$, so $k_{2}=2$.

6. Now $k_{1}+k_{2}=7=n$, and $d p_{v_{2} v_{5}}\left(v_{i}\right)=0 ; \forall v_{i} \notin S_{1}$. Therefore $D=S_{1}=\left\{v_{2}, v_{5}\right\}$ is a minimum dominating set and $\gamma=\left|S_{1}\right|=2$.

\section{MAIN RESULTS}

\subsection{Theorem}

If $S$ be the set of all supports of a connected graph $G$, then domination number $\gamma \geq|S|$.

Proof: Let $G$ be a connected graph of order $\mathrm{n}$ and $S$ be the set of all supports in $G$ such that $|S|=k$. Then there are atleast $k$ pendent vertices in $G$. Pendent vertices are dominated by either support or by itself. Therefore either each support or corresponding pendent vertex must be in dominating set $D \geq k$. Thus $\gamma \geq k=|S|$.

\subsection{Theorem}

If $I$ be the set of all internal vertices of a tree $T$ then connected domination number $\gamma_{c}=|I|$.

Proof: Let $T$ be a tree of order $n$ and let $|I|=m$ that is there are $m$ internal vertices in $T$. Obviously $\langle I\rangle$ is a sub tree of $T$ and $|\bar{N}(I)|=n$. Hence $I$ is a connected dominating set of a tree $T$.

$I$ is the smallest connected dominating set of tree $T$. If it is not so, let $I$ ' be another connected dominating set of tree $T$ such that $I^{\prime} \subset I$. Clearly $\exists$ at least one $v_{i} \in I$ such that $v_{i} \notin I^{\prime}$.

Now two cases arise:

(i) When $v_{i} \in I$ and $v_{i}$ is a support of $T$ such that $v_{i} \notin I^{\prime}$. In this case $\left|\bar{N}\left(I^{\prime}\right)\right|<n$, so $I^{\prime}$ is not a dominating set of tree $T$.

(ii) When $v_{i} \in I$ and $v_{i}$ is not a support of $T$ such that $v_{i} \notin I^{\prime}$. Clearly in this case $\left\langle I^{\prime}\right\rangle$ is not connected.

Thus there exist no proper subset of $I$ which is connected dominating set for $T$. Hence $I$ is the smallest connected dominating set for $T$ and $\gamma_{c}=|I|$.

\subsection{Example}

Consider the Fig. 4 of labelled tree with 13 vertices as given below:

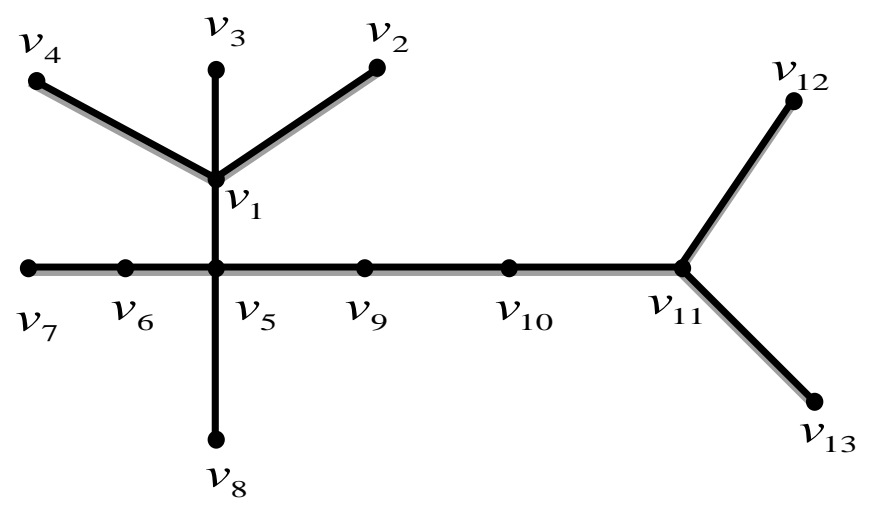

Fig 4: Labelled tree with six internal vertices and seven pendent vertices

Here $I=\left\{v_{1}, v_{6}, v_{5}, v_{9}, v_{10}, v_{11}\right\}$ and $\gamma_{c}=6=|I|$.

\section{In the next lemma a relation between connected domination number and domination number for a tree has been established.}

\subsection{Lemma}

If domination number $\gamma$ of a tree $T$ is equal to number of support, then

$$
\gamma_{c}=\gamma+k,
$$

where $k$ is the number of internal vertices which are not support.

Proof: Let $S$ be the set of all support of a tree $T$ such that domination number $\gamma=|S|$. Let $k$ be the number of internal vertices of $T$ which are not support then $\gamma_{c}=$ number of internal vertices

$\gamma_{c}=$ number of support + number of internal vertices which are not support.

$$
\begin{aligned}
& \gamma_{c}=|S|+k \\
& \gamma_{c}=\gamma+k . \quad(\because \gamma=
\end{aligned}
$$$$
|S|)
$$

\subsection{Example}

In the following tree $\gamma=3, \gamma_{c}=4$, and $v_{2}$ is only internal vertex which is not support that is $k=1$ which verify the above result

$$
\gamma_{c}=\gamma+k
$$




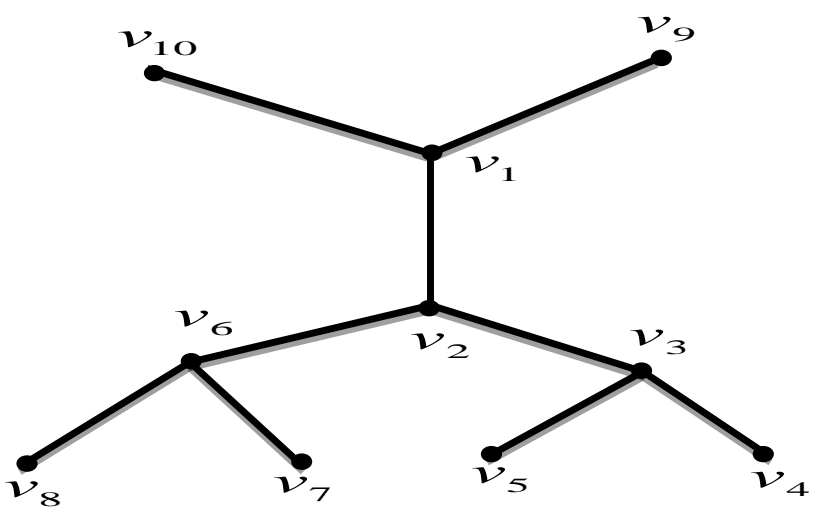

Fig 5: Tree with one internal vertex without support

\subsection{Corollary}

If every internal vertex of a tree is support, then domination number and connected domination number of the tree are same.

Proof: Consider a tree $T$ with domination number $\gamma$ and connected domination number $\gamma_{c}$. Let $k$ be the internal vertices but not support then $\gamma_{c}=\gamma+k$.

Since every internal vertex of $T$ is support that is there is no internal vertex which is not support therefore $k=0 \Rightarrow \gamma_{c}=\gamma$.

Note: This corollary is also proved by Arumugam et al [2] as a theorem.

\subsection{Example}

Consider the tree in Fig.-6 as given below:

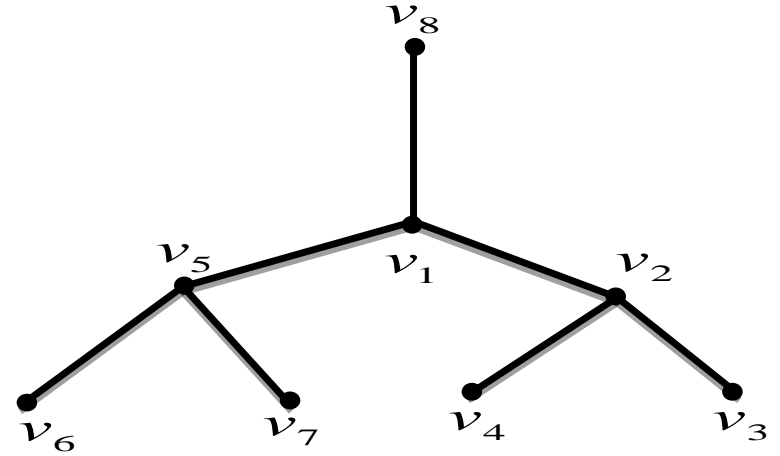

Fig 6: Tree with all internal vertices are support

Here $v_{1}, v_{2}$ and $v_{5}$ are three internal vertices which all are support and

$$
\gamma=3=\gamma_{c}
$$

\subsection{Theorem}

If $T$ be a tree with $m$ pendent vertices then independence number $\beta$ of $T$ is $m+l$, where $l$ is maximum number of internal vertices such that $\forall v_{i}, v_{j} \in I-S$ and $v_{i}$ and $v_{j}$ are not adjacent.

Proof: Let $E$ be the largest set of independent vertices of a tree $T$ such that $p_{1}, p_{2}, p_{3}, \ldots, p_{m}$ are $m$ pendent vertices, $s_{1}, s_{2}, s_{3}, \ldots, s_{t}$ are $t$ supports and $i_{1}, i_{2}, i_{3} \ldots, i_{k}$ are $k$ internal vertices except support of $T$, then $m \geq t$. Since each pendent vertex is adjacent only support and $m \geq t$. Therefore $\forall p_{i} \in E ; \quad 1 \leq i \leq m$ and $\forall s_{i} \notin E ; \quad 1 \leq i \leq t$.

Now out of $k$ internal vertices which are not support let maximum $l$ internal vertices are independent to each other. Suppose that these vertices are $i_{1}, i_{2}, i_{3}, \ldots, i_{l}$ then $\forall i_{j} \in E ; \quad 1 \leq j \leq l$. $E=\left\{p_{1}, p_{2}, p_{3}, \ldots, p_{m} ; i_{1}, i_{2}, i_{3}, \ldots, i_{l}\right\}$ is the largest set of independent vertices for $T$ and hence

$$
\beta=|E|=m+l \text {. }
$$

\subsection{Example}

In Fig. $4 ; v_{2}, v_{3}, v_{4}, v_{7}, v_{8}, v_{12}, v_{13}$ are pendent vertices that is $m=7$ and $v_{9}, v_{10}$ are two internal vertices except support but these two vertices are adjacent so $l=1$ and

$$
\beta=8=m+l \text {. }
$$

\subsection{Corollary}

If every internal vertex is support in a tree $T$, then independence number $\beta$ of $T$ is equal to number of pendent vertices in $T$.

Proof: Let $T$ be a tree with $m$ pendent vertices then independence number of $T$ is given by

$$
\beta=m+l,
$$

where $l$ is maximum number of internal vertices such that $\forall v_{i}, v_{j} \in I-S$ and $v_{i}$ and $v_{j}$ are not adjacent. Since every internal vertex is support therefore $l=0$ that is $\beta=m=$ number of pendent vertices.

\subsection{Example}

Consider the tree in Fig.-6.Here $v_{1}, v_{2}$ and $v_{5}$ are three internal vertices which all are support and $v_{3}, v_{4}, v_{6}, v_{7}$ and $v_{8}$ are 5 pendent vertices that is $m=5$ and $\beta=5=m$ which verify the result.

In the next theorem, a relation among domination number, connected domination number and independence number for a tree has been established.

\subsection{Theorem}

If in a tree $T$, every internal vertex except support are independent to each other then

$$
\beta+\gamma=\gamma_{c}+m \text {. }
$$

Proof: Let $T$ be a tree such that every internal vertex except support are independent to each other than by result of corollary 4.6

$$
\gamma_{c}=\gamma+k
$$

where $k$ is the number of internal vertices which are not support. By result of theorem 4.8,

$$
\beta=m+l,
$$


where $l$ is maximum number of internal vertices such that $\forall v_{i}, v_{j} \in I-S$ and $v_{i}$ and $v_{j}$ are not adjacent. Since every internal vertex except support are independent to each other therefore $k=l$, using in equation (2) gives

$$
\beta=m+k \Rightarrow k=\beta-m
$$

using equation (3) in equation (1) gives

$$
\gamma_{c}=\gamma+\beta-m \Rightarrow \beta+\gamma=\gamma_{c}+m \text {. }
$$

\section{ACKNOWLEDGEMENT}

This research work is supported by Gurukula Kangri University, Haridwar (UK), India. The authors are grateful to Dr. S. R. Verma for his valuable suggestions to improve the manuscript of this paper.

\section{REFERENCES}

[1] Harary, F., 1997 Graph Theory, Narosa Publishing House.
[2] Arumugam, S., Joseph, J. P. 1999 On graphs with equal domination and connected domination numbers, Discrete Mathematics vol.206, 45-49.

[3] Deo, N. 2005 Graph Theory with Applications to Engineering and Computer Science, Prentice-Hall of India Private Limited.

[4] Saoud, M., Jebran, J. 2009 Finding A Minimum Dominating Set by Transforming Domination of Vertices, Acta Universitatis Apulensis 19.

[5] Hedetniemi, S.T., Laskar, R.C. 1990 Bibliography on Domination in Graph and Some Basic Definitions on domination Parameters, Discrete Mathematics vol.86, 257-277. 